\title{
Interactomic study on interaction between lipid droplets and mitochondria
}

\author{
Jing $\mathrm{Pu}^{1,2^{*}}$, Cheol Woong $\mathrm{Ha}^{3 *}$, Shuyan Zhang ${ }^{1}$, Jong Pil Jung ${ }^{3}$, Won-Ki Huh ${ }^{3 凶}$, Pingsheng Liu ${ }^{1 凶}$ \\ ${ }^{1}$ National Laboratory of Biomacromolecules, Institute of Biophysics, Chinese Academy of Sciences, Beijing 100101, China \\ ${ }^{2}$ Graduate University of Chinese Academy of Sciences, Beijing 100101, China \\ ${ }^{3}$ School of Biological Sciences, Research Center for Functional Cellulomics, Institute of Microbiology, Seoul National University, \\ Seoul 151-747, Republic of Korea \\ $\triangle$ Correspondence: pliu@ibp.ac.cn (P. Liu), wkh@snu.ac.kr (W. -K. Huh) \\ Received April 22, 2011 Accepted May 22, 2011
}

\begin{abstract}
An increasing body of evidence shows that the lipid droplet, a neutral lipid storage organelle, plays a role in lipid metabolism and energy homeostasis through its interaction with mitochondria. However, the cellular functions and molecular mechanisms of the interaction remain ambiguous. Here we present data from transmission electron microscopy, fluorescence imaging, and reconstitution assays, demonstrating that lipid droplets physically contact mitochondria in vivo and in vitro. Using a bimolecular fluorescence complementation assay in Saccharomyces cerevisiae, we generated an interactomic map of protein-protein contacts of lipid droplets with mitochondria and peroxisomes. The lipid droplet proteins Erg6 and Pet10 were found to be involved in $75 \%$ of the interactions detected. Interestingly, interactions between 3 pairs of lipid metabolic enzymes were detected. Collectively, these data demonstrate that lipid droplets make physical contacts with mitochondria and peroxisomes, and reveal specific molecular interactions that suggest active participation of lipid droplets in lipid metabolism in yeast.
\end{abstract}

KEYWORDS peroxisomes, bimolecular fluorescence complementation assay, protein-protein interaction, lipid metabolism, Erg6

\section{INTRODUCTION}

Lipid droplets are spherical organelles existing in many cell types and consisting of a core of neutral lipids and a coating of a monolayer of phospholipids with peripheral as well as embedded proteins (Murphy, 2001; Tauchi-Sato et al., 2002). It was considered an inert particle for energy storage until recent years when increasing evidence showed that lipid droplets are highly dynamic and are able to interact with other organelles (Martin and Parton, 2006; Goodman, 2008; Murphy et al., 2009; Zhang et al., 2010). In particular, lipid droplets were found to physically contact the ER, mitochondria, endosomes and peroxisomes, although the physiological function of these contacts remains unknown. Studies of lipid droplet dynamics will pave the way for a better understanding of the cellular network for energy and mass transfer (particularly lipids) as well as the pathology of metabolic diseases.

Lipid droplets are frequently seen attached to mitochondria in adipocytes (Novikoff et al., 1980; Blanchette-Mackie and Scow, 1983) and hepatocytes (Kalashnikova and Fadeeva, 2006). In skeletal muscle cells, association of lipid droplets with mitochondria has also been observed (Shaw et al., 2008) and found to become more abundant when energy requirements increase due to physical exercise (Tarnopolsky et al., 2007). Lipases such as hormone-sensitive lipase (HSL) (Egan et al., 1992) and adipose triglyceride lipase (ATGL) (Zimmermann et al., 2004) have been found in lipid droplets, demonstrating a localized capacity for mobilizing energy stores. The product of lipase activity, free fatty acids, is the substrate for $\beta$-oxidation in the mitochondrion and/or peroxisome, suggesting that an interaction between these organelles is related to energy utilization. Besides energy transfer, lipid trafficking may be another purpose of an interaction between lipid droplets and mitochondria. In yeast, $\beta$-oxidation occurs in peroxisomes, but physical contacts between lipid

"These authors contributed equally to the work. 
droplets and mitochondria were still seen, and many mitochondrial proteins were found to be localized in lipid droplets (Binns et al., 2006). These findings indicate that the interaction between lipid droplets and mitochondria may be involved in multiple physiological events and may be well regulated. Additionally, it has been reported that lipid droplets can move in a microtubule- and dynein-dependent manner (Guo et al., 2005). The SNARE protein synaptosome associated protein 23 (SNAP23) was reported to mediate the interaction between lipid droplets and mitochondria (Jägerström et al., 2009), although the molecular mechanism remains unknown.

In the present study, we focused on protein-protein interactions to search for mediators of the association between lipid droplets and mitochondria by bimolecular fluorescence complementation (BiFC) assay in Saccharomyces cerevisiae. BiFC enables direct visualization of protein-protein interactions in living cells, based on the formation of a fluorescent complex of two non-fluorescent fragments of a fluorescent protein. Interaction between a bait and a prey protein, each fused to one of these fragments, brings the fragments into close contact, resulting in a fluorescent signal. BiFC is an effective and straightforward tool to study protein-protein interactions, avoiding the possibility of non-physiological protein interactions caused by cell lysis and mixing the contents of different cellular compartments. Many protein-protein interactions have been detected successfully in different cell types and organisms using BiFC assays (Hu and Kerppola, 2003; Brasaemle et al., 2004; Blondel et al., 2005). Furthermore, in yeast, proteins being examined by BiFC are expressed under the control of their native promoters (Sung and Huh, 2007). Here, we present data demonstrating the existence of contact between lipid droplets and mitochondria in skeletal muscle tissue and cells by morphology studies and in vitro reconstitution assays. Using a BiFC assay we studied the interactomics of lipid droplets and mitochondria and peroxisomes in S. cerevisiae and detected 116 protein-protein interactions.

\section{RESULTS}

\section{Contact between lipid droplets and mitochondria}

Skeletal muscle cells are one of the major energy consumers in the animal body and the interaction between lipid droplets and mitochondria probably plays a key role in energy homeostasis. Previous morphological studies have revealed that lipid droplets and mitochondria might physically contact each other in muscle tissues (Shaw et al., 2008). Consistent with this, we observed a close association between mitochondria and lipid droplets in rat skeletal muscle tissue by transmission electron microscopy (TEM). Interestingly, some lipid droplets and mitochondria appeared to share portions of the membrane as a boundary (Fig. 1A, left panel, arrow). In other cases, lipid droplets and mitochondria were closely associated but retained independent membranes (Fig. 1A; right panel, arrow). To verify the interaction in living cells, we treated cells of the rat skeletal muscle cell line L6 with oleate for $3 \mathrm{~h}$ to induce the formation of droplets. After washing off the oleate, the cellular positions of mitochondria and lipid droplets were determined by confocal microscopy and 3D reconstruction. Consistent with TEM results, lipid dropletmitochondrion contact was frequently observed (Fig. 1B; lower panel, arrow), and statistical analysis revealed that the contact rate was correlated with lipid droplet size (data not shown). These data suggest that lipid droplet-mitochondrion interactions are dynamic.

To further verify mitochondrion-lipid droplet interaction, an in vitro reconstitution assay using purified lipid droplets and mitochondria was performed as described previously (Liu et al., 2008). SDS-PAGE analysis of the isolated fractions (Fig. 1C; upper panel) demonstrated distinct protein profiles of purified lipid droplets (LD), mitochondria (Mito), postnuclear supernatant (PNS) and cytosol (cyto), suggesting that lipid droplets and mitochondria were highly enriched. After incubation with mitochondria, lipid droplets were isolated from the reaction system, and their surface proteins were analyzed by Western blot. Translocase of the inner mitochondrial membrane 23 (Tim 23), a mitochondrial inner membrane protein, was used as a mitochondrial marker, and adipocyte differentiation-related protein (ADRP) as a lipid droplet protein loading control. While Tim 23 could not be detected (Fig. 1C; lower panel, lane 1) in the proteins obtained from purified lipid droplets, those obtained from lipid droplets after incubation with mitochondria contained Tim 23 (lane 2), suggesting that mitochondria interacted with lipid droplets during incubation. When GTPys or isolated cytosol was added to the reaction system, stronger signals of Tim 23 were detected (lanes 3 and 4), which suggests that GTPYs or cytosol stimulated the mitochondrion-lipid droplet interaction. ATP was found to have no effect on the interaction (data not shown). These in vitro assays offered further evidence that lipid droplets and mitochondria can interact with each other, and some physiological factors such as GTP and some cytosolic component(s) may regulate their interactions.

\section{Screening the proteins involved in the lipid droplet-mitochondrion (or peroxisome) interaction by BiFC assay in S. cerevisiae}

To explore the proteins involved in the organelle interaction, we took advantage of BiFC assays in S. cerevisiae to analyze interactions between proteins found in our organelles of interest in vivo. The $\mathrm{C}$-terminus of the fluorescence protein Venus (VC) was fused to the lipid droplet proteins, and the Nterminus (VN) to the mitochondrial proteins. We expected fluorescence signals to be generated by the fusion of $\mathrm{VC}$ and VN if the proteins interacted with each other (Fig. 2A). Since, 
A

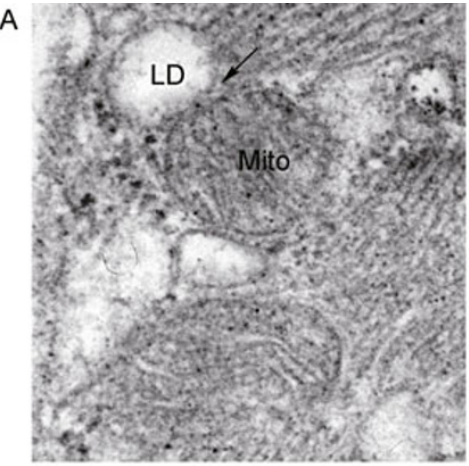

B

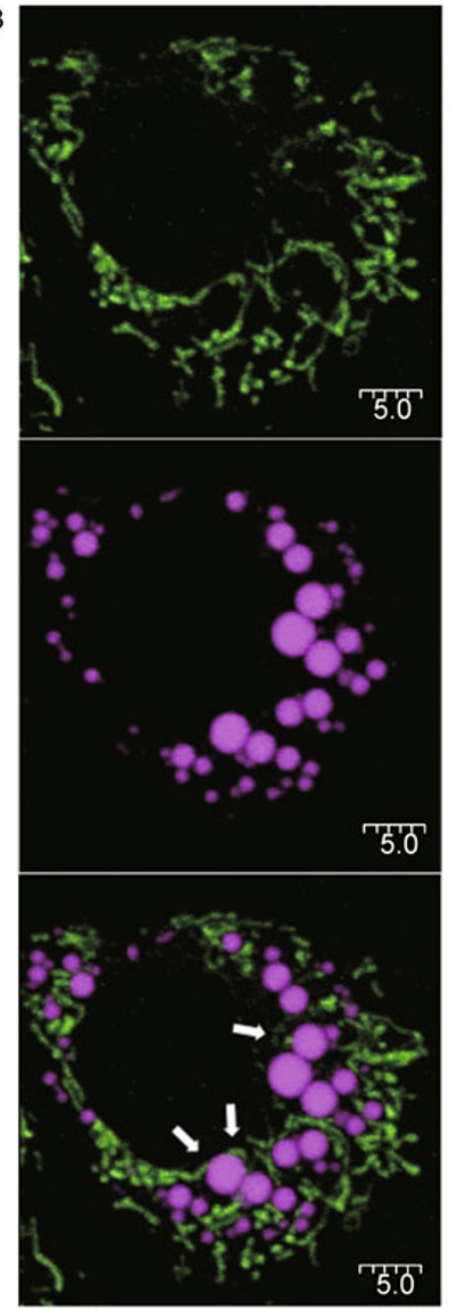

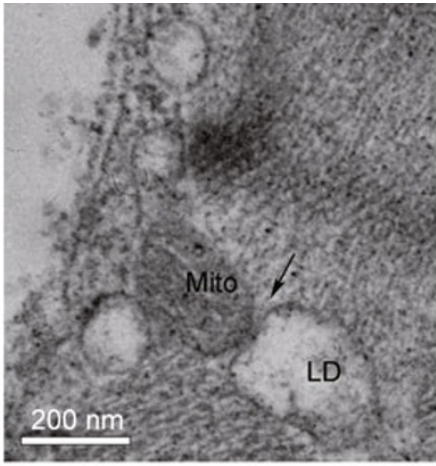

C

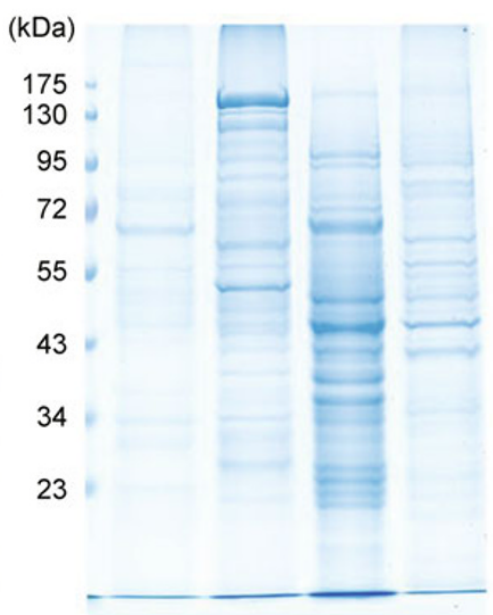

M LD Mito PNS Cyto
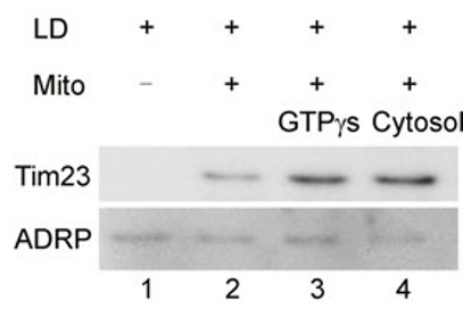

Figure 1. Interaction between mitochondria and lipid droplets in skeletal muscle cells. (A) The ultrastructure of rat skeletal muscle tissue was obtained by transmission electron microscopy (TEM). Mito, mitochondrion; LD, lipid droplet. Bar $=200 \mathrm{~nm}$. (B) $\mathrm{L6}$ cells were treated with $200 \mu \mathrm{mol} / \mathrm{L}$ oleate in the growth medium for $12 \mathrm{~h}$ and maintained in the growth medium for $3 \mathrm{~h}$ after washing oleate off. Mitochondria (upper panel) and lipid droplets (middle panel) were stained with MitoTracker Green FM and LipidTOX Deep Red, respectively. Fluorescence images were obtained using a confocal microscope and 3D reconstructed by software FLUOVIEW. The contact between lipid droplets and mitochondria were determined by 3D reconstruction movie and indicated by arrows (lower panel). Bar $=5 \mu \mathrm{m}$. (C) Proteins were extracted from purified lipid droplets (LD), mitochondria (Mito), cytosol (Cyto) and postnuclear supernatant (PNS) from C57 mouse liver tissues and were resolved by $10 \%$ SDS-PAGE. The gel was stained with a Colloidal Blue Staining Kit (upper panel). M, molecular weight marker. Purified lipid droplets and mitochondria were subjected to in vitro reconstitution assays. GTPYs (lane 3) or isolated cytosol (lane 4) was added to the reaction system. Total proteins of lipid droplets were isolated from the system and were analyzed by immunoblotting with the indicated antibodies (lower panel). 
A

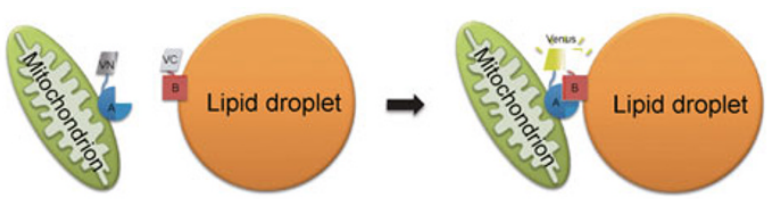

B

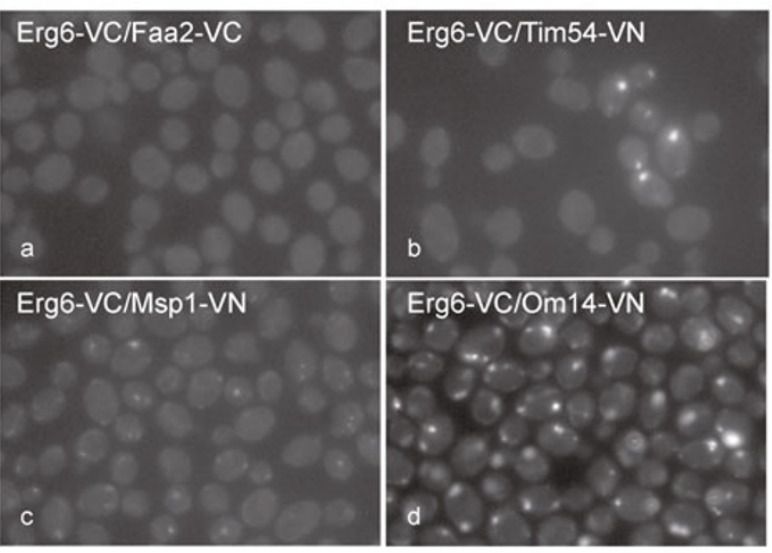

C

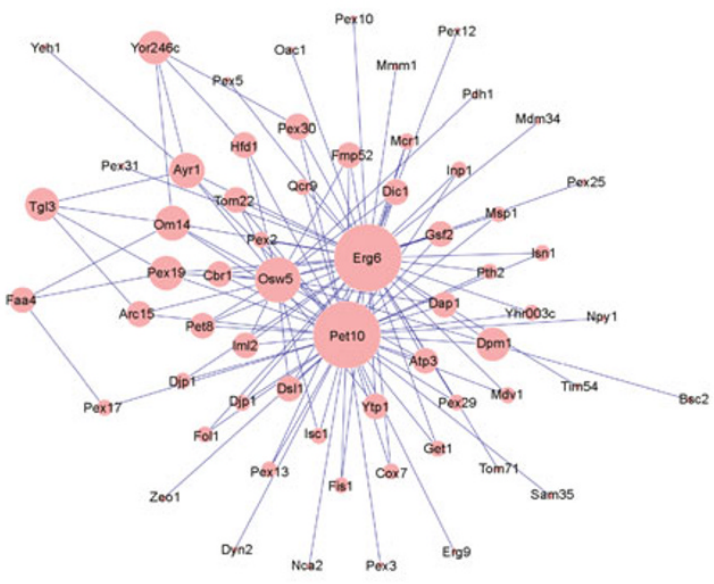

D

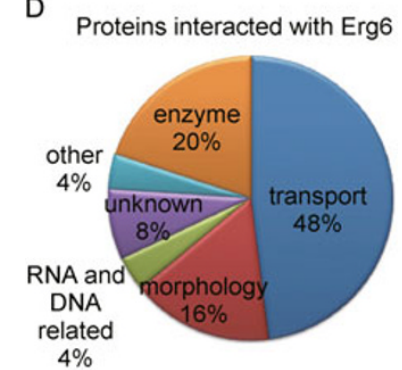

Proteins interacted with Pet10

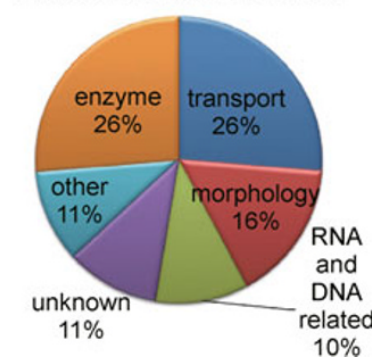

Figure 2. Proteins involved in the organelle interaction. (A) The schema of BiFC assay probing lipid droplet and mitochondrion interaction. The interaction between $\mathrm{C}$-terminally $\mathrm{VN}$-tagged mitochondrial protein $\mathrm{A}$ and $\mathrm{C}$-terminally $\mathrm{VC}$-tagged lipid droplet protein $B$ brings $V C$ and $V N$ into close proximity resulting in a fluorescent signal. (B) The BiFC assay was performed and signals from fluorescence complementation were monitored by fluorescence microscopy. Diploid yeast cells expressing C-terminally VC-tagged Tgl3 and Faa2 were analyzed for BiFC and used as a negative control (a). The fluorescence images with 3 different signal patterns are shown in b, $c$ and d. The two genes C-terminally tagged with VC or VN are indicated as gene X-VC/gene Y-VN. (cC) The proteinprotein interaction network visualized by Cytoscape. The node size was according to node degree. (D) The proteins interacting with Erg6 (upper panel) or Pet10 (lower panel) were catalogued by their function referring to SGD and the percentages of each category are indicated. 
unlike mammalian cells, yeasts carry out $\beta$-oxidation in peroxisomes, we included peroxisomal proteins as well in our BiFC assays. We selected 22 lipid droplet proteins as baits and 225 mitochondrial and peroxisomal proteins as preys for our screen. We chose 22 lipid droplet proteins, 81 mitochondrial proteins, and 21 peroxisomal proteins based on their localizations as reported in the Yeast GFP Fusion Localization database (Huh et al., 2003). An additional 103 proteins of the outer membrane of mitochondria and 20 peroxisomal proteins were chosen from the Saccharomyces genome database (SGD).

In yeast cells, the number and size of lipid droplets vary depending on cell growth. To obtain robust BiFC signals, the yeast cells were cultured in YPD medium and inoculated into SC medium for fluorescence detection when growing in the logarithmic phase. Compared with cells grown in the YPD medium, cells grown in SC medium displayed reduced autofluorescence (Fig. S1A; right panel vs left panel) and increased lipid droplet formation (Fig. S1B; right panel vs left panel).

We found 116 protein pairs that yielded positive signals in our BiFC assays. They included 8 lipid droplet proteins, 37 mitochondrial proteins and 19 peroxisomal proteins (Tables 1 and 2). There are 52 pairs of protein-protein interactions corresponding to interactions between lipid droplets and mitochondria (or peroxisomes) listed in Table 1. Table 2 displays interactions detected between proteins for which there is conflicting or ambiguous localization information in the two databases. In some cases, this may be due to the fact that the proteins have multiple localizations. In our experiments, the BiFC signals appeared in a punctate pattern, consistent with localization to lipid droplets. However, the signal intensity, number of puncta and localization patterns varied (Fig. 2Bb-d). This hints at a complex interplay between these organelles. Two VC-tagged strains were mated as a negative control and no fluorescence was observed in these cells (Fig. 2Ba).

\section{The interaction between lipid droplet and mitochondrion (or peroxisome) may be important for lipid metabolism}

Among the 22 lipid droplet proteins examined, 8 were found to interact with prey proteins. Of these 8 proteins, Erg6 and Pet10 were the most active, interacting with many mitochondrial and peroxisomal proteins and generating $75 \%$ of the total positive signals. The network of the interactions was visualized by Cytoscape (Shannon et al., 2003), wherein node degree was mapped to node size (Fig. 2C). Furthermore, besides mitochondrial and peroxisomal proteins, Erg6 interacted with 10 other lipid droplet proteins including 2 enzymes of sterol biosynthesis, 2 enzymes of triglyceride metabolism and 6 with unknown function (Table 3). Interestingly, Pet10 is one of these 10 lipid droplet proteins that interact with Erg6. Therefore, it is possible that Erg6 and
Table 1 Protein-protein interactions identified by BiFC assay in S. cerevisiae

\begin{tabular}{|c|c|c|c|c|c|c|}
\hline \multirow{2}{*}{ Gene } & \multicolumn{6}{|c|}{ Lipid droplet proteins } \\
\hline & Erg6 & Tgl3 & Faa4 & Pet10 & Yor246c & Osw5 \\
\hline Pdh1 & & & & & & + \\
\hline Fol1 & $+^{a}$ & & & + & & \\
\hline Nca2 & & & & + & & \\
\hline Yhr003c & + & & & + & & \\
\hline Sam35 & & & & + & & \\
\hline Mcr1 & + & & & + & & \\
\hline Pth2 & + & & & + & & \\
\hline Om14 & + & + & + & + & + & + \\
\hline Mdv1 & + & & & + & & \\
\hline Msp1 & + & & & + & & \\
\hline Atp3 & + & & & + & & + \\
\hline Pet8 & + & & & + & & + \\
\hline Qcr9 & + & & & + & & \\
\hline Cox7 & + & & & + & & \\
\hline Mdm34 & + & & & & & \\
\hline Tom71 & + & & & & & \\
\hline Mmm1 & + & & & & & \\
\hline Tim54 & + & & & & & \\
\hline Oac1 & + & & & & & \\
\hline Pex30 & + & & & + & + & \\
\hline Inp1 & + & & & + & & \\
\hline Pex3 & & & & + & & \\
\hline Pex13 & + & & & + & & \\
\hline Pex2 & + & & & + & & \\
\hline Pex17 & & & + & + & & \\
\hline Pex5 & + & & & & & \\
\hline Pex25 & + & & & & & \\
\hline Pex12 & + & & & & & \\
\hline Pex10 & + & & & & & \\
\hline Total & 24 & 1 & 2 & 19 & 2 & 4 \\
\hline
\end{tabular}

${ }^{\mathrm{a}} \mathrm{BiFC}$ signal positive; upper panel, mitochondrial proteins; lower panel, peroxisomal proteins.

Pet10 constitute a "reaction core" in lipid droplets. We categorized the mitochondrial and peroxisomal proteins that interact with Erg6 or Pet10 according to their functions. The pie charts in Fig. 2D show that most of the proteins that interact with Erg6 (upper panel) or Pet10 (lower panel) are either enzymes or proteins involved in transport, implying that lipid droplets might function primarily in metabolism and trafficking.

Three of the several pairs of interacting proteins identified in our assays are involved in lipid metabolism: Erg6/Mcr1, Tgl3/Ayr1, and Tgl3/Pex11 (Table 4). Erg6 is an enzyme involved in ergosterol synthesis and converts zymosterol to fecosterol by methylating at position C- 24 . It is interesting that 
Table 2 Protein-protein interactions identified by BiFC assay in S. cerevisiae (supplement)

\begin{tabular}{|c|c|c|c|c|c|c|c|c|c|c|}
\hline \multirow{2}{*}{ Gene } & \multicolumn{8}{|c|}{ Lipid droplet proteins } & \multicolumn{2}{|c|}{ Localization } \\
\hline & Erg6 & Tgl3 & Yeh1 & Faa4 & Pet10 & Bsc2 & Yor246c & Osw5 & SGD & GFP \\
\hline Arc15 & $+^{a}$ & + & & & + & & & & mito $^{b}$ & Actin \\
\hline Jsn1 & + & & & & + & & & & mito & Ambiguous \\
\hline Tom22 & + & & & & + & & & + & mito & Ambiguous \\
\hline Iml2 & + & & & & + & & & + & mito & cyto $^{c} n u^{d}$ \\
\hline $\mathrm{Hfd} 1$ & & & & & + & & + & + & mito & endo $^{e} L^{f}$ \\
\hline Cbr1 & + & & & & + & & & & mito & ER \\
\hline Ayr1 & + & + & + & & + & & + & + & mito & ER \\
\hline Fmp52 & + & & & & + & & & + & mito & ER \\
\hline Erg9 & & & & & + & & & & mito & ER \\
\hline Gsf2 & + & & & & + & & & + & mito & ER \\
\hline Dpm1 & + & & & & + & + & & + & mito & ER \\
\hline Dic1 & + & & & & + & & & + & mito & ER \\
\hline Get1 & + & & & & + & & & & mito & ER \\
\hline Zeo1 & & & & & + & & & & mito & ER, peri ${ }^{9}$ \\
\hline Dap1 & + & & & & + & & & + & mito & ER, cyto \\
\hline Isc1 & & & & & + & & & & mito & $\mathrm{No}^{\mathrm{h}}$ \\
\hline Ytp1 & + & & & & + & & & + & mito & No \\
\hline Fis1 & + & & & & + & & & & mito/pero ${ }^{\mathrm{i}}$ & Ambiguous \\
\hline Pex31 & + & & & & & & & & pero & Ambiguous \\
\hline Pex32 & + & & & & & & & & pero & Ambiguous \\
\hline Pex29 & + & & & & + & & & & pero & $\begin{array}{l}\text { Ambigu- } \\
\text { ous, }\end{array}$ \\
\hline Npy1 & & & & & + & & & & pero & cyto, nu \\
\hline Pex19 & + & + & & + & + & & & + & pero & cyto \\
\hline Dsl1 & + & & & & + & & & + & pero & ER \\
\hline Dyn2 & & & & & + & & & & pero & ER \\
\hline Djp1 & + & & & & + & & & & pero & ER \\
\hline Total & 20 & 3 & 1 & 1 & 24 & 1 & 2 & 12 & & \\
\hline
\end{tabular}

${ }^{\mathrm{a} B i F C}$ signal positive; ${ }^{\mathrm{b}}$ mitochondrion; ${ }^{\mathrm{c}}$ cytosol; ${ }^{\mathrm{d}}$ nucleus; ${ }^{\mathrm{e}}$ endosome; flipid droplet; ${ }^{\mathrm{g}}$ cell periphery; ${ }^{\mathrm{h}}$ no localization scored for ORF; iperoxisome.

Mcr1, which is also an enzyme involved in ergosterol biosynthesis, is localized to the mitochondria and was found in our assay to interact with Erg6. It is possible that lipid droplets and mitochondria separately complete parts of the ergosterol synthetic process, and then deliver or exchange metabolites between them via physical interaction to complete ergosterol biosynthesis. Similarly, fatty acids released from triglycerides might be hydrolyzed by $\mathrm{Tgl} 3$ in lipid droplets and feed into Ayr1-involved phosphatidic acid synthesis in mitochondria or Pex11-involved $\beta$-oxidation in peroxisomes. These protein-protein interactions suggest that lipid droplets might both offer substrates to or accept substrates from lipid metabolic processes occurring in mitochondria or peroxisomes and complete the metabolism via physical interactions with them. It would be economical for cells to generate lipids by using metabolic intermediates delivered by lipid droplets rather than by de novo synthesis, and our data provide support for this model.

\section{DISCUSSION}

In mammalian cells, fatty acid oxidation mainly occurs in mitochondria, but the majority of cellular fatty acids come from the lipolysis of triglycerides in lipid droplets. It is possible that fatty acids might be transferred from lipid droplets to mitochondria via direct physical interactions between these organelles. Lipid droplet proteomic studies carried out in several species, tissues, and cell lines such as yeast (Binns et al., 2006), plant cells (Katavic et al., 2006), Drosophila (Beller et al., 2006; Cermelli et al., 2006), liver (Turróet al., 2006), 3T3-L1 cells (Brasaemle et al., 2004), and CHO cells (Liu et al., 2004) have identified hundreds of proteins 
Table 3 Lipid droplet proteins interacting with Erg6

\begin{tabular}{lll}
\hline Name & Molecular function & Biological process \\
\hline Erg7 & Lanosterol synthase activity & Ergosterol biosynthetic process \\
Tgl4 & Sterol esterase activity & Triglyceride catabolism \\
& Triglyceride lipase activity & Triglyceride mobilization \\
Yeh1 & Sterol esterase activity & Sterol metabolism \\
Tgl3 & Triglyceride lipase activity & Triglyceride catabolic process; Cellular lipid metabolic process \\
Coy1 & Unknown & Golgi vesicle transport \\
Yor246c & Oxidoreductase activity & Unknown \\
Pet10 & Unknown & Aerobic respiration \\
Rrt8 & Unknown & Unknown \\
Bsc2 & Unknown & Unknown \\
Ymr148w & Unknown & Unknown \\
\hline
\end{tabular}

Table 4 Protein-protein interactions involved in lipid metabolism between lipid droplets and mitochondria or peroxisomes

\begin{tabular}{|c|c|c|c|c|c|}
\hline \multicolumn{3}{|c|}{ Lipid droplet protein (bait) } & \multicolumn{3}{|c|}{ Mitochondrial or peroxisomal protein (prey) } \\
\hline Name & Molecular function & Biological process & Name & Molecular function & Biological process \\
\hline Erg6 & $\begin{array}{l}\text { Sterol } \\
24-C-m e t h y \mid t r a n s f e r a s e\end{array}$ & $\begin{array}{l}\text { Ergosterol } \\
\text { biosynthetic process }\end{array}$ & Mcr1 & Cytochrome b5 reductase & $\begin{array}{l}\text { Ergosterol biosynthetic } \\
\text { process }\end{array}$ \\
\hline Tgl3 & $\begin{array}{l}\text { Triglyceride lipase } \\
\text { activity }\end{array}$ & $\begin{array}{l}\text { Triglyceride } \\
\text { catabolic process } \\
\text { Cellular lipid } \\
\text { metabolic process }\end{array}$ & Ayr1 & $\begin{array}{l}\text { Acylglycerone-phosphate } \\
\text { reductase }\end{array}$ & $\begin{array}{l}\text { Phosphatidic acid } \\
\text { biosynthetic }\end{array}$ \\
\hline & & & Pex11 & Unknown & Fatty acid oxidation \\
\hline
\end{tabular}

localized in lipid droplets. Interestingly, a variety of mitochondrial proteins were frequently identified in isolated lipid droplets in proteomic studies (Table S1), suggesting either dual localization or physical association of these mitochondrial proteins with those of lipid droplets. For instance, the mitochondrial protein NADH-cytochrome b5 reductase 3 was found in isolated lipid droplets in several species (Table S1). In our study, this protein was found to interact with lipid droplet proteins Erg6 and Pet10 (Table 2; Cbr1). This and other interactions identified in our study provide a mechanistic explanation for the presence of mitochondrial proteins in lipid droplets reported in previous studies.

Almost every cellular event is mediated by networks of protein-protein interactions. Interactions of lipid droplet proteins with each other or with those of other cellular compartments are likely essential for lipid droplet functions. Additionally, interactions between specific protein pairs might subserve organelle-to-organelle contact. Unfortunately, the $\mathrm{BiFC}$ assay cannot distinguish between interactions involved in organelle contact from those related to other cellular functions. However, the properties of the proteins can provide us some important clues.

Previous studies have reported that ergosterols function as regulators of membrane permeability and fluidity (Gaber et al., 1989). Additionally, increased sterol concentrations promote membrane fusion (Tedrick et al., 2004). Based on these results from previous studies and our results, we can speculate that lipid droplets move along the cell skeleton to contact mitochondria, leading to physical and functional coupling mediated by lipid droplet and mitochondrial proteins. Erg6, acting along with other enzymes in lipid droplets, and mitochondrial enzymes together, completes ergosterol synthesis. Increased ergosterol concentrations could alter the properties of the membrane and consequently make the membrane microenvironment suitable for protein movement. This could then allow exchange of proteins between the organelles.

What proteins might initiate the junction between organelles? Several studies have reported that proteins that regulate membrane trafficking such as Rabs and SNAREs mediate the interactions between lipid droplets and other organelles (Zehmer et al., 2009). Rab proteins are generally known as GTPase switches controlling membrane trafficking among intracellular compartments. Multiple Rab proteins were identified in lipid droplets in a proteomic study by Liu et al. (Liu et al., 2004), and Liu and his coworkers later reported that Rabs are capable of regulating the interaction between lipid droplets and early endosomes (Liu et al., 2007). Like other GTPases, Rabs have two conformations: an inactive form bound to GDP and an active form bound to 
GTP. Our results show that GTP can enhance interactions between lipid droplets and mitochondria (Fig. 1C, lower panel), suggesting that GTPases may be involved in the interaction and Rabs may be the mediators. In our BiFC assay, the Rab5 yeast homolog Vsp21 generated a positive signal with Erg6, but C-terminally VN-tagged Vsp21 was also able to interact with cytosolic free VC fragment, so the possibility of false positive could not be excluded. The resolution of this issue awaits future work.

Among the proteins we assayed by BiFC, there were 4 membrane trafficking proteins in lipid droplets-Use1, Snx41, Coy1 and Sso1, and 3 proteins in mitochondrial outer membrane-Mdm10, Mmm1 and Vsp21. However, these proteins were not found to interact with each other, and only Mmm1 was found to interact with Erg6. Although this result was surprising, a likely explanation is that any interactions among these proteins, if present, could be transient in nature. Besides Erg6 and Pet10, another lipid droplet protein Osw5 was also found to interact with many mitochondrial and peroxisomal proteins (Tables 1 and 2), accounting for about $14 \%$ of the total positive signals. As SNARE proteins, which mediate membrane fusion, contain cytosolic alpha helices that drive membrane interaction, interestingly, Osw5 is predicted to contain an $\mathrm{N}$-terminal transmembrane domain (Fig. S1C; upper panel) and a C-terminal a helix (Fig. S1C; lower panel). It is plausible that this a helix is invovled in membrane interaction in a manner similar to those of SNARE proteins, binding to partners present in mitochondria or peroxisomes. Its partner in mitochondrion side might be Tom22, a mitochondrial outer membrane protein. Similar to Osw5, Tom22 is predicted to contain a transmembrane segment (Fig. S1D; upper panel) and a complex cytosolic domain (Fig. S1D; lower panel) that is thought to act as a receptor for precursor proteins. Therefore, Osw5 and Tom22 might be the pair of proteins linking lipid droplets and mitochondria by protein-protein interaction. Another mitochondrial protein, Om14, was also found to be very active in our assay, interacting with 6 lipid droplet proteins including 3 enzymes and 3 proteins of unknown function (Table 1). Om14 is an integral mitochondrial outer membrane protein with 3 helical transmembrane segments. Its interaction with Erg6 (Fig. 2B, panel d) yielded a very strong BiFC signal and, as such, Om14 is another candidate for further investigation.

\section{MATERIALS AND METHODS}

\section{Materials}

Oleate, tricine, GTPYs and concanavalin A were purchased from Sigma-Aldrich (St. Louis, MO). MitoTracker Green FM, LipidTOX Deep Red, Colloidal Blue Staining Kit, a-MEM and FBS were from Invitrogen (Carlsbad, CA). Yeast extract, peptone, yeast nitrogen base, agar and antibodies against Tim 23 were from BD Biosciences (San Jose, CA). Uranyl acetate, $25 \%$ glutaraldehyde solution (EM grade) and lead citrate were all from Electron Microscopy Sciences
(Pennsylvania, USA). Osmium tetraoxide (EM grade) was purchased from Nakalai Tesque Co. (Kyoto, Japan). Quetol 812 was purchased from Nisshin EM Co., Ltd. (Tokyo, Japan).

\section{Cell culture}

L6 cells were maintained in a-minimal essential medium ( $\alpha-M E M)$ supplemented with $10 \%(\mathrm{v} / \mathrm{v}) \mathrm{FBS}, 100 \mathrm{U} / \mathrm{mL}$ penicillin, and $100 \mathrm{U} / \mathrm{mL}$ streptomycin at $37^{\circ} \mathrm{C}$ with $5 \% \mathrm{CO}_{2}$.

\section{Transmission electron microscopy}

The ultrastructure of rat skeletal muscles was examined by ultra-thin sectioning and transmission electron microscopy (TEM). After collection, male Sprague-Dawley rat muscle tissues were cut into small pieces and then prefixed in $2.5 \%(\mathrm{w} / \mathrm{V})$ glutaraldehyde in PBS $(\mathrm{pH} 7.4)$ at $4^{\circ} \mathrm{C}$ for 2 days and postfixed in $2 \%(w / v)$ osmium tetraoxide at room temperature for $1.5 \mathrm{~h}$. The fixed samples were dehydrated by incubation in an ascending concentration series of ethanol $(35 \%, 50 \%, 70 \%, 90 \%, 95 \%$ and $100 \%)$. After embedding in Quetol 812, samples were prepared as $90 \mathrm{~nm}$ sections with Leica EM UC6 Ultramicrotome (Leica company). Each section was stained with $2 \%(w / v)$ uranyl acetate for $15 \mathrm{~min}$ and then with lead citrate for $5 \mathrm{~min}$. The stained sections were examined under a FEI Tecnai20 (FEI Company) electron microscope.

\section{Cell fluorescence imaging}

L6 cells were treated with $200 \mu \mathrm{mol} / \mathrm{L}$ oleate in the growth medium for $12 \mathrm{~h}$, then washed with pre-warmed medium 3 times, maintained in the growth medium for $3 \mathrm{~h}$, and incubated in the growth medium containing $25 \mathrm{nmol} / \mathrm{L}$ MitoTracker Green FM and LipidTOX Deep Red (11000 diluted) at $37^{\circ} \mathrm{C}$ for $30 \mathrm{~min}$. Fluorescence signals were captured by z-axis scanning with a confocal microscope at both the $488 / 525 \mathrm{~nm}$ and $630 / 660 \mathrm{~nm}$ excitation/emission wavelength channels. Three-dimensional (3D) reconstruction and animation was completed using the FLUOVIEW software.

\section{In vitro reconstitution assay}

Lipid droplet purification was performed as previously described (Liu et al., 2008). The pellet from lipid droplet purification was washed twice and resuspended in $1 \mathrm{~mL}$ buffer $\mathrm{B}(20 \mathrm{mmol} / \mathrm{L}$ HEPES, $\mathrm{pH} 7.4$, $100 \mathrm{mmol} / \mathrm{L} \mathrm{KCl}, 2 \mathrm{mmol} / \mathrm{L} \mathrm{MgCl}_{2}$ ) for mitochondrion purification. The pellet-resuspended mixture was loaded on the top of a Percoll step gradient ( $2 \mathrm{~mL} 80 \%, 4.5 \mathrm{~mL} 52 \%$, and $4.5 \mathrm{~mL} 26 \%$ ) and centrifuged at $40,000 \mathrm{~g}$ for $45 \mathrm{~min}$ at $4^{\circ} \mathrm{C}$. The visible interface between $26 \%$ and $52 \%$ Percoll was collected and washed 3 times with buffer $B$ to remove Percoll, yielding purified mitochondria.

The cytosol was isolated from the postnuclear supernatant (PNS) fraction in the lipid droplet purification steps. The PNS was centrifuged at $200,000 \mathrm{~g}$ for $45 \mathrm{~min}$ at $4^{\circ} \mathrm{C}$, and the cytosol was collected between lipid droplets on the top and the membrane pellet on the bottom.

Purified lipid droplets and mitochondria were mixed with or without $1 \mathrm{mmol} / \mathrm{L}$ GTPys or cytosol and incubated at $37^{\circ} \mathrm{C}$ for $30 \mathrm{~min}$ and mixed every $5 \mathrm{~min}$. After incubation, lipid droplets and mitochondria were separated by centrifugation at $20,000 \mathrm{~g}$ for $3 \mathrm{~min}$ at $4^{\circ} \mathrm{C}$. 
Mitochondria (pellet) and the buffer were removed, and lipid droplets were resuspended with cold buffer $B$. This step was repeated twice. Lipid droplet proteins were extracted by acetone and dissolved in $2 \times$ sample buffer for Western blot analysis using the indicated antibodies. All animal experiments were approved by the Institutional Animal Care and Use Committee and followed the Animal Use Guidelines of the Institute of Biophysics, Chinese Academy of Sciences, Beijing.

\section{BiFC assay}

A single-step, PCR-based approach was applied to tag the C-termini of yeast proteins with yellow fluorescent protein fragments for the BiFC assay (Sung and Huh, 2007). Yeast strains BY4741 (MATa his $3 \Delta 1$ leu2 $\Delta 0$ met $15 \Delta 0$ ura $3 \Delta 0$ ) and BY4742 (MATa his $3 \Delta 1$ leu2 $\Delta 0$ lys $2 \Delta 0$ ura3 $\triangle 0$ ) were maintained at $30^{\circ} \mathrm{C}$ in the YPD medium. The PCR product of $\mathrm{N}-(\mathrm{VN})$ or C-terminal fragment (VC) of Venus, a variant of yellow fluorescent protein, was obtained using pFA6a-VNHis3MX6 or pFA6a-VC-kanMX6 as template. Yeast transformation was performed using the lithium acetate method. The strains of VCtagged lipid droplet proteins derived from BY4742 were mated with the strains of $\mathrm{VN}$-tagged mitochondrial proteins derived from BY4741. The resulting diploid yeast cells were cultured in the synthetic complete (SC) medium to mid-logarithmic phase, transferred to 96well glass-bottomed microplates coated with concanavalin $A$ and analyzed by fluorescence microscopy. The BiFC signals were observed using a Zeiss Axiovert 200M inverted microscope with a Plan-NeoFluar 100×/1.3 NA oil immersion objective. Fluorescence images were taken using a standard fluorescein isothiocyanate filter set (excitation band pass filter, $450-490 \mathrm{~nm}$; beam splitter, $510 \mathrm{~nm}$; emission band pass filter, $515-565 \mathrm{~nm}$ ).

\section{Protein databases}

Protein localization information was derived from the following databases:

Yeast GFP Fusion Localization database, http://yeastgfp.yeastgenome.org

Saccharomyces genome database, http://www.yeastgenome.org/

\section{ACKNOWLEDGEMENTS}

The authors would like to thank Dr. John Zehmer for his critical reading and useful suggestions. The authors also want to thank Dr. Huina Zhang for her administrative support. This work was supported by grants from the National Basic Research Program of China (Grant Nos. 2009CB919000 and 2010CB833703), the National Natural Science Foundation of China (Grant Nos. 30871229 and 30971431), and the 21C Frontier Functional Proteomics Project (FPR08A1-060) funded by the Ministry of Education, Science and Technology, Republic of Korea.

\section{ABBREVIATIONS}

ADRP, adipocyte differentiation-related protein; BiFC, Bimolecular Fluorescence Complementation; PNS, postnuclear supernatant; TEM, transmission electron microscopy; Tim 23, inner mitochondrial membrane 23; VC, C-terminal fragment of Venus; VN, N-terminal fragment of Venus
Supplementary material is available in the online version of this article at http://dx.doi.org/10.1007/s13238-011-1061-y and is accessible for authorized authors.

\section{REFERENCES}

Beller, M., Riedel, D., Jänsch, L., Dieterich, G., Wehland, J., Jäckle, H., and Kühnlein, R.P. (2006). Characterization of the Drosophila lipid droplet subproteome. Mol Cell Proteomics 5, 1082-1094.

Binns, D., Januszewski, T., Chen, Y., Hill, J., Markin, V.S., Zhao, Y., Gilpin, C., Chapman, K.D., Anderson, R.G., and Goodman, J.M. (2006). An intimate collaboration between peroxisomes and lipid bodies. J Cell Biol 173, 719-731.

Blanchette-Mackie, E.J., and Scow, R.O. (1983). Movement of lipolytic products to mitochondria in brown adipose tissue of young rats: an electron microscope study. J Lipid Res 24, 229-244.

Blondel, M., Bach, S., Bamps, S., Dobbelaere, J., Wiget, P., Longaretti, C., Barral, Y., Meijer, L., and Peter, M. (2005). Degradation of Hof1 by SCF(Grr1) is important for actomyosin contraction during cytokinesis in yeast. EMBO J 24, 1440-1452.

Brasaemle, D.L., Dolios, G., Shapiro, L., and Wang, R. (2004). Proteomic analysis of proteins associated with lipid droplets of basal and lipolytically stimulated 3T3-L1 adipocytes. J Biol Chem 279, 46835-46842.

Cermelli, S., Guo, Y., Gross, S.P., and Welte, M.A. (2006). The lipiddroplet proteome reveals that droplets are a protein-storage depot. Curr Biol 16, 1783-1795.

Egan, J.J., Greenberg, A.S., Chang, M.K., Wek, S.A., Moos, M.C. Jr, and Londos, C. (1992). Mechanism of hormone-stimulated lipolysis in adipocytes: translocation of hormone-sensitive lipase to the lipid storage droplet. Proc Natl Acad Sci U S A 89, 8537-8541.

Gaber, R.F., Copple, D.M., Kennedy, B.K., Vidal, M., and Bard, M. (1989). The yeast gene ERG6 is required for normal membrane function but is not essential for biosynthesis of the cell-cyclesparking sterol. Mol Cell Biol 9, 3447-3456.

Goodman, J.M. (2008). The gregarious lipid droplet. J Biol Chem 283, 28005-28009.

Guo, Y., Jangi, S., and Welte, M.A. (2005). Organelle-specific control of intracellular transport: distinctly targeted isoforms of the regulator Klar. Mol Biol Cell 16, 1406-1416.

$\mathrm{Hu}$, C.D., and Kerppola, T.K. (2003). Simultaneous visualization of multiple protein interactions in living cells using multicolor fluorescence complementation analysis. Nat Biotechnol 21, 539-545.

Huh, W.K., Falvo, J.V., Gerke, L.C., Carroll, A.S., Howson, R.W., Weissman, J.S., and O'Shea, E.K. (2003). Global analysis of protein localization in budding yeast. Nature 425, 686-691.

Jägerström, S., Polesie, S., Wickström, Y., Johansson, B.R., Schröder, H.D., Højlund, K., and Boström, P. (2009). Lipid droplets interact with mitochondria using SNAP23. Cell Biol Int 33, 934-940.

Kalashnikova, M.M., and Fadeeva, E.O. (2006). Ultrastructural study of liver cells from rooks living in ecologically unfavorable areas. Izv Akad Nauk Ser Biol (2), 133-141.

Katavic, V., Agrawal, G.K., Hajduch, M., Harris, S.L., and Thelen, J.J. (2006). Protein and lipid composition analysis of oil bodies from two Brassica napus cultivars. Proteomics 6, 4586- 4598.

Liu, P., Bartz, R., Zehmer, J.K., Ying, Y., and Anderson, R.G. (2008). 
Rab-regulated membrane traffic between adiposomes and multiple endomembrane systems. Methods Enzymol 439, 327-337.

Liu, P., Bartz, R., Zehmer, J.K., Ying, Y.S., Zhu, M., Serrero, G., and Anderson, R.G. (2007). Rab-regulated interaction of early endosomes with lipid droplets. Biochim Biophys Acta 1773, 784 793.

Liu, P., Ying, Y., Zhao, Y., Mundy, D.I., Zhu, M., and Anderson, R.G. (2004). Chinese hamster ovary K2 cell lipid droplets appear to be metabolic organelles involved in membrane traffic. J Biol Chem 279, 3787-3792.

Martin, S., and Parton, R.G. (2006). Lipid droplets: a unified view of a dynamic organelle. Nat Rev Mol Cell Biol 7, 373-378.

Murphy, D.J. (2001). The biogenesis and functions of lipid bodies in animals, plants and microorganisms. Prog Lipid Res 40, 325-438.

Murphy, S., Martin, S., and Parton, R.G. (2009). Lipid dropletorganelle interactions; sharing the fats. Biochim Biophys Acta 1791, 441-447.

Novikoff, A.B., Novikoff, P.M., Rosen, O.M., and Rubin, C.S. (1980). Organelle relationships in cultured $3 \mathrm{~T} 3-\mathrm{L} 1$ preadipocytes. J Cell Biol 87, 180-196.

Shannon, P., Markiel, A., Ozier, O., Baliga, N.S., Wang, J.T., Ramage, D., Amin, N., Schwikowski, B., and Ideker, T. (2003). Cytoscape: a software environment for integrated models of biomolecular interaction networks. Genome Res 13, 2498-2504.

Shaw, C.S., Jones, D.A., and Wagenmakers, A.J. (2008). Network distribution of mitochondria and lipid droplets in human muscle fibres. Histochem Cell Biol 129, 65-72.

Sung, M.K., and Huh, W.K. (2007). Bimolecular fluorescence complementation analysis system for in vivo detection of proteinprotein interaction in Saccharomyces cerevisiae. Yeast 24,
767-775.

Tarnopolsky, M.A., Rennie, C.D., Robertshaw, H.A., FedakTarnopolsky, S.N., Devries, M.C., and Hamadeh, M.J. (2007). Influence of endurance exercise training and sex on intramyocellular lipid and mitochondrial ultrastructure, substrate use, and mitochondrial enzyme activity. Am J Physiol Regul Integr Comp Physiol 292, R1271-R1278.

Tauchi-Sato, K., Ozeki, S., Houjou, T., Taguchi, R., and Fujimoto, T. (2002). The surface of lipid droplets is a phospholipid monolayer with a unique Fatty Acid composition. J Biol Chem 277, 44507-44512.

Tedrick, K., Trischuk, T., Lehner, R., and Eitzen, G. (2004). Enhanced membrane fusion in sterol-enriched vacuoles bypasses the Vrp1p requirement. Mol Biol Cell 15, 4609-4621.

Turró, S., Ingelmo-Torres, M., Estanyol, J.M., Tebar, F., Fernández, M. A., Albor, C.V., Gaus, K., Grewal, T., Enrich, C., and Pol, A. (2006). Identification and characterization of associated with lipid droplet protein 1: A novel membrane-associated protein that resides on hepatic lipid droplets. Traffic 7, 1254-1269.

Zehmer, J.K., Huang, Y., Peng, G., Pu, J., Anderson, R.G., and Liu, P. (2009). A role for lipid droplets in inter-membrane lipid traffic. Proteomics 9, 914-921.

Zhang, S., Du, Y., Wang, Y., and Liu, P. (2010). Lipid Droplet - A Cellular Organelle for Lipid Metabolism. Acta Biophisica Sinica 26, 97-105.

Zimmermann, R., Strauss, J.G., Haemmerle, G., Schoiswohl, G., Birner-Gruenberger, R., Riederer, M., Lass, A., Neuberger, G., Eisenhaber, F., Hermetter, A., et al. (2004). Fat mobilization in adipose tissue is promoted by adipose triglyceride lipase. Science 306, 1383-1386. 